\title{
SUPPORTING ELDERLY LIVING LONGER AT HOME: A FRAMEWORK FOR BUILDING A SUSTAINABLE ECO-SYSTEM
}

\author{
Linda Askenäs and Jan Aidemark \\ Linnaeus University, Universitetsplatsen 1, 35195, Växjö; Sweden
}

\begin{abstract}
Long term success of eHealth initiatives rests on the cooperation between major parties in society. This paper reports on an ongoing project with the aim of providing support for elderly living longer at home. The general template of Quadruple Helix cooperation aids the formation and execution of projects with broad impact ambitions and could guide the design of solutions for an aging population. A general model for cooperation, based on the different challenges encountered in an ongoing research and development project, is presented and discussed, with regard to a process of the Quadruple Helix type. Key components of the processes include a lifecycle-based process view of the solutions and the technical base, comprising the continuous development of solutions, business models and technical platforms. At the centre of the approach reside user interaction aspects and the desired outcomes of changed behaviours. These rest on the abilities of a technical platform to afford the personalization of solutions fitting the varying needs of elderly living longer at home. As a result, a lifecycle-based and end-user driven co-design process is presented as an example of a Quadruple Helix project organization. The project shows a way of operationalizing Quadruple Helix in software development through the use of co-design concepts.
\end{abstract}

\section{KEYWORDS}

Living Longer at Home, IoT Platform, Quadruple Helix, Cooperative Design

\section{INTRODUCTION}

An aging population poses many challenges to a society where a novel use of information technologies can be part of the solution. The creation of viable eHealth services which are flexible, user-oriented and affordable is a challenge. This report provides the outlines of a cooperative framework for the continued design, development and sustainable deployment of IT-based services for elderly living longer at home. Although living longer at home is desired by the elderly, there are problems connected with factors such as disease, declining general health or the home environment. As these problems can be expected to be highly personal and changing over time, any effort of supporting the longer at home concept must be equally adaptable and flexible to meet these needs. There is an obvious problem concerning static vertical solutions coming from different technical spheres and creating a complex and expensive support situation. This research reports on a current project aiming at working with this problem situation by providing a framework which enables organizations from different areas of business, technical development, public services and user collectives to cooperate and, in a learning spirit, continuously co-create new technical and organizational solutions through working within a general Quadruple Helix framework. However, the operationalization of Quadruple Helix is still not clearly explored (Höglund and Linton, 2017). Here we look at an example connecting "Quadruple Helix" innovation environments with IT-based innovation and co-design as a way of creating cooperation between experts from different areas, with a focus on end-users as experts on their own situations.

The research objective of the paper is to explore the setup, processes, structures and technical capabilities of an eHealth ecosystem as it emerges in an ongoing project. This is formulated as a three-set framework which will continue to guide the sustained development over time by providing a stability of core platform services, common ways of working with core processes and the creation of novel solutions by an extended business network. The paper concludes with an integrating model, where the Quadruple Helix concept is realized by co-operative design efforts throughout a software life-cycle model, with end-user involvement as its guiding principle. 


\section{RESEARCH BACKGROUND}

\subsection{Living Longer at Home}

The fact that elderly people wish to live longer at home is illustrated, for example, by a US study (ARRP, 2004) showing that $83 \%-86 \%$ of people over 65 wish to go on staying in their own homes. Living at home could become problematic for many reasons such as poor wellbeing, dependent living, fall risk, chronic disease, dementia, social isolation, depression and medication management (Sixsmith and Sixsmith, 2008). Efforts are extant to support the elderly and mitigate the problems by finding innovative solutions for remote communication and monitoring, as well as home modifications (Graybill et al., 2914). Some of these could be achieved or made easier and cheaper by using information-based solutions, including sensors and end-user applications in combination with traditional solutions (Roberts et al., 2012). They also offer an opportunity to work more proactively by facilitating the adaptation to new circumstances and by preserving abilities and reaching a self-understanding of limitations and possibilities as well as finding new solutions for coping in everyday life (Khosravi, \& Ghapanchi, 2016).

Current home care efforts could guide the understanding of the conditions of home support by using more technology preventively, before traditional people home care becomes necessary. "Living longer at home" could include a large set of solutions, for example fall prevention systems aimed at supporting relatively healthy elderly persons still living at home to form part of a larger "living longer at home" scenario. The systems could, for example, help them to avoid the problems caused by serious fall accidents, which in turn lead to decreased physical fitness and an increasing risk of further falls (Hamm et al. 2016). Hospitalization, increased societal costs and a decreased standard of life for the elderly are consequences that need to be avoided. A possible number of IT systems could offer support by including a wide variety of problem prevention measures with the focus on creating learning and a behaviour cycle change.

Experiences from home care organizations show some conditions for what constitutes effective support for the elderly. It is important to work through personalization and to take care of their potential, making their situation and its possibilities steer the support (Turjamaa et al. 2014).

\subsection{Quadruple Helix Logic}

The "Quadruple Helix", including public organization, users/civil society, business and research/education, provides a framework for how to understand and foster innovative environments. It incorporates the perspective of the "media-based and culture-based" public (Carayannis and Campbell, 2009). This extension, compared to Triple Helix, creates the conditions for an innovation ecosystem that is suitable for the current and still emerging knowledge economy and society.

In this kind of knowledge economy, described by Carayannis and Campbell (2009) as fractal knowledge, there is a great need of fostering and making accommodation for the co-existence and co-evolution of different perspectives of knowledge residing in the four different strains of the helix. What is needed in such a knowledge system, Carayannis and Campbell (2009) argue, is the "adaptive capacity to combine and integrate different knowledge and innovation modes via co-evolution, co-specialisation and co-opetition" of the fragmented knowledge stock of an eco-system. These are all aspects of the co-operational ability of the Quadruple Helix environment, stressing the key resource of knowledge for business success and societal development.

Working in a Quadruple Helix model entails an increased complexity (Ivanova, 2014) which could be more difficult to achieve. Ivanova (2014) points to the importance of using more advanced communication technologies in Quadruple Helix projects, due to the greater complexity of interactions between innovation actors. Many more dimensions, such as the local-global one, can be added to an innovation system (Leydesdorff, 2012), thereby increasing its complexity but also its potency. The increased focus on the end user brings greater variation and adds to the number of parties involved in an innovation process. The Quadruple Helix entails a turn towards user-driven innovation (Miller et al. 2016), where it is the end user who creates a demand and could become a driver for innovation in such projects. With its fourth strain (the end-user), Quadruple Helix provides a "dimension of democracy" (Carayannis and Campbell, 2014, and makes space for open knowledge creation, based on broader strands of society. 


\subsection{Cooperative Design}

Co-design (CD), participative design (PD) and human-centred design (HCD) are all examples of approaches for design and development that lay focus on the context in a broad sense, as well as on different groups of people involved in the situation of using new systems or processes. The involvement of stakeholders is assumed to be a key component in successful development and change projects. The goal is to achieve a better understanding of the social and cultural situation that the design process is based on, with the intention of improving the outcome of the design process. Suchman describes the area as having a focus on the "... human, creative, and effective relationship between those involved in technology's design and its use, and in that way between technology and the human activities that provide technological systems with their reason for being" (Suchman, 1993). In addition to extracting requirements from the user community and validating solutions, higher demands are made on co-designing. Co-designing is more than a technique for engaging users in the development process. The focus on the "co" element in co-design should be understood as a cooperation between the parties engaged in the development that gives room for and respect to the different sets of perspectives, needs and competencies that each party brings to the process of creating, for example, new and innovative information system solutions. Co-design is the process of end users, experts and technical developers designing together for the effective use of combined expertise and creativity throughout the design life cycle (Sanders \& Stappers, 2008). Its core is to create and share knowledge about the project, including problems and solutions from the different perspectives of the parties involved (Ref. Anonymised). Co-design is a co-learning process that creates a common understanding of the different perspectives that the groups of people involved bring to the project.

The co-design idea has also been applied on a grander scale, where co-creation has been discussed as a means for transforming cities (Mulder, 2018; Foth, 2018). In the context of city planning, Mulder (2018) argues that "co-creative partnerships can play a crucial role in societal change".

\section{RESEARCH APPROACH}

\subsection{Method Discussion}

The research is based on an ongoing innovation and product development project applying a case study logic to generalize findings about how to organize and structure projects of this type. The case study approach aims at an in-depth investigation of a current phenomenon within its real-life context (Stake, 1995). Stake argues that the single case is important through its uniqueness as well as its possibilities for finding communalities and connections to wider phenomena. A descriptive stance is taken, providing a structured account of how a project aims at solving challenges in eHealth areas by means of a Quadruple Helix structure. The focus of the study is a single project, which "aims at understanding the dynamics present within single settings" (cf. Eisenhardt, 1989). The project primarily consists of technical and business staff from commercial companies and of professionals from the health care sector. The role of academic researchers in the project is to support and facilitate the interactions between the different perspectives, especially related to the socio-technical dimension and to the way users are involved in the development project. This research stance comprises the roles of generating research results and of contributing to business-related outcomes and is akin to participative action research (Chevalier and Buckles, 2013).

\subsection{Project Description}

The project FRONT-VL, is focused on the problems connected to the trends of longer lives and fewer children, which is creating a situation today with fewer people in working age. This demographic transition is viewed as one of the major challenges to European economies and welfare systems. The approach to solutions is concentrated on smart and efficient technical solutions to increase possibilities for the elderly to live at home without being dependent on children or in-home care. By enabling elderly people to live at home - either independently or assisted - for as long as possible, a good quality of life can be maintained while at the same time reducing care costs. Based on a number of practical user situations, end-user services will be defined and 
developed to support the end user with ICT relevant to all stakeholders. The use of state-of-the-art machine learning and big data analysis methodologies, together with a profound IoT-based data acquisition, will allow the development of sophisticated predictive health-related services. This supports the analysis and interpretation of data of individuals on a big scale. The consortium is international, being composed of organizations from all strands of the "Quadruple Helix", including private companies, universities, governmental and end-user organizations representing public interests.

\section{CASE STUDY: “LIVING LONGER AT HOME" ECO SYSTEM}

The project needs to facilitate constant changes in a flexible manner, accommodating those of people in different phases of their lives. In order to realize the possibilities of long-term and continued innovation, there is a need for a model where different logics can reside, interact, thrive and cooperate. The key abilities and resources are identified as partner interactions, technical platforms and business models, joined by a cooperative development model. All the identified aspects stress the need of corporations in the process of development, as a technical capacity and for the creation of business opportunities.

\subsection{Organizational Perspective: Continuous Development Processes}

Success rests on the integration and cooperation between different logics of the user, the technician and business, all in the context of the wider societal realities and visions of changing behaviours, needs and wants. This is not only a design but an ongoing design, development and implementation process sustained over time. The organizational and processual knowledge guiding the continuous development is in itself a core aspect of the solution. A number of processes have been defined:

The general framework process frames the project on a conceptual level and coordinates the main technical development process. The core platform is expected to be enhanced, as new end user services are created and deployed. Here we find the overall path of the project with strategic directions and coordination between technical, user, service and market perspectives.

Co-design processes develop and integrate knowledge from all main processes, generic framework, agile co-design and service development, into an understanding of requirements for the outcomes of the project, both from technical and business perspectives. The co-sensing process provides the technical input and specification for technical development. The co-design process is the venue between the competencies from all the different processes, where the meeting between different knowledge areas can create a common understanding. This means that knowledge is created for technical development, service development and market strategies as well as forming a meeting place between the professional developers, business and technology, on the one hand, with the different user groups, such as end users and professionals, on the other.

- $\quad$ Service development entails that the software is, in the end, a product or service that needs to be structured and packaged.

- $\quad$ To-market involves the creation of strategies and methods for bringing the service to an intended market.

There are several important interconnections between the different processes and their tasks. These are all key elements in the knowledge-creating and sharing environment of a Quadruple Helix system.

- Developers learning about users. The technical developers involved work iteratively, testing and advancing the technical ideas during the co-design. It is not only a question of providing the requirements the developers need to get a sense of the essence of use situations and of the actual users. Developing tools for an elderly population might be rather complex, as the social and cultural gap between user community and developers is wider than usual. The users have different abilities and also form a relatively fragmented group which is to cover most of the population without excluding minor groups.

- User understanding of technology. This means creating an awareness of the overall technical limitations and restrictions in the generic framework and standards. Teaching end users about technical possibilities creates a class of super users who are able to provide input to the system's requirements of higher quality and relevance. 
- Combining business and technical development. Combining technical excellence with the creation of a viable service/product on the market makes the interconnections between the business development and technical development important. It is necessary to reach an understanding of who is willing to pay, which are the actors involved on the market and of what solutions are coming or already existing on the market that can be used in the design, be partnered up with or be treated as a competitor.

- Piloting and to-market strategy. When the design is in its piloting phase, the possibilities of how to supply it to the customers will become part of the co-design with the different participants. The implementation of a technical support process will have to be installed and evaluated at the same time to achieve the design of a service change program and a maintenance service.

\subsection{Technical Perspective: Reference Architecture and Technical Platform}

At the core of a project, a reference architecture, including a service platform, connects all aspect of project operations from a technical and integrational perspective. The architecture is structured in six layers, connecting everything from use cases and business models down to sensors and data capture. The core of the architecture is a technical service platform based on which applications (combinations of end user interfaces and sensor systems) can be developed. Data management and data integration will be unified and standardized throughout all the applications employed for their greater usefulness and benefit to an elderly person. This ensures the possible integration of data for analyses across all platform applications.

- $\quad$ Layer 1 comprises the devices and sensors, currently defining 3 different types, characterized by integration and data capturing and transport.

Layer 2 involves the IoT data integration. Sensors and data sources residing in this layer provide a massive amount of usage value and document data. By the IoT integration platform, the data integrates, collects and transports the data storage system of Layer 3. The data integration engine is what enables IoT and the use of multiple data sources by various technologies and integration methodologies.

- Layer 3 constitutes the data management platform. The "Continua Health Alliance" (http://www.pchalliance.org/) is applied to manage the seamless sharing of data from the pre-certified Continua products and solutions in the platform. The main goal of utilizing Continua is to ensure that the devices and services can interoperate across borders and in accordance with the security and privacy requirements already adopted worldwide via the Continua Design Guidelines (CDG). This enables us to run a testing event by using tools, codes and resources developed by Continua.

- Layer 4 concerns data abstraction (API Layer). This means the reduction of a particular body of data in order to achieve a simplified representation of the whole. In other words, what this layer does is removing some characteristics from the data captured in the previous layers in order to reduce it to a set of essential ones.

- Layer 5 is the application layer containing end user interaction interfaces which provide information services such as reports, statistics, graphics and aggregation based on sensor data or derived by analytical means.

- $\quad$ Layer 6 includes clients and processes, i.e., end-users and business processes making practical use of the data provided by the platform. 


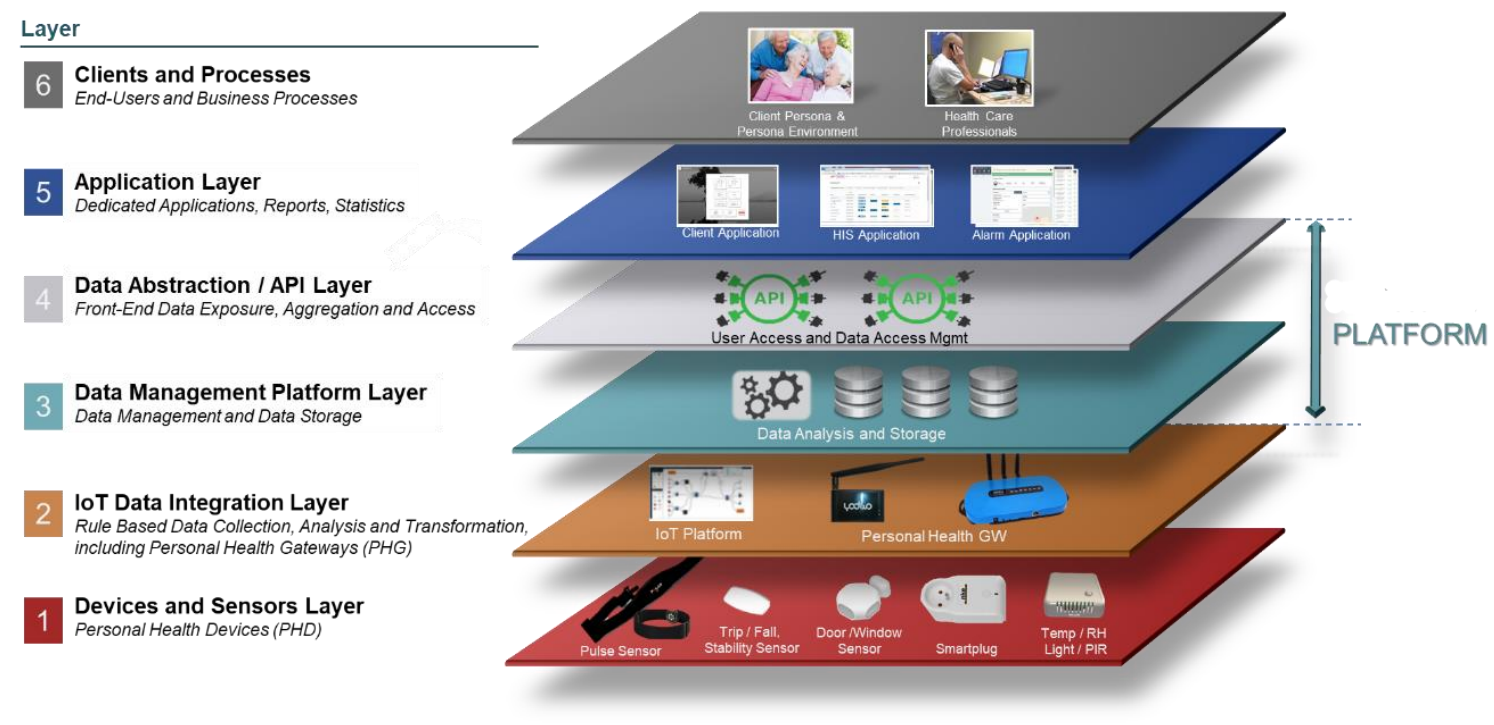

Figure 1. Reference architecture and platform

\subsection{Applications Perspective: End-User Solutions}

As part of the effort of the project is to define and develop the reference architecture, a set of new applications (or existing ones integrated into the platform) forms a core example of the "living longer at home" concept. These are continuously developed through co-design, focusing on the interaction between the different parties of experts (e.g., technicians, users, or business representatives). What takes place within the framework ensures the integration of the different applications offering the possibility of making a joint analysis of data and arriving at a holistic understanding of the situation of the elderly. These are the applications included in the project so far (with more to follow):

- Fall prevention, a feedback system from body or environmental sensors, alerting users to or advising them about risks of fall as well as suggesting preventive actions. Self-understanding and acquiring an insight into real behaviour and actual hazardous ways of conducting everyday actions will provide a basis for changes in behaviour to a safer way of leading everyday life.

- A rehabilitation training system monitoring the movements of video-based exercises. The basic setup is a computer with the training software, a Kinect camera and a TV set which enables the elderly to perform basic training programs while helping them to avoid fall accidents. The feedback that provides information on whether the exercises are correctly performed makes it into a system possible for the elderly to use on their own at home.

- VR-base instruction and a learning system for fall prevention information. Using a VR headset, the elderly are introduced to various home situations where they are to detect fall hazards and make a choice of how to tackle them. This learning environment provides a novel approach towards engaging elderly persons to changing their mindset and behaviour in everyday situations.

- Mental training games. Online games, developed as a mental wellness toolset for self-usage for various categories of elderly people. The games provide help to measure and visualize mental changes as well as tendencies of how mental capabilities are evolving, all in an entertaining way. While playing various games (such as solitaire, whack-a-mole or memory), the performance of the elderly is measured and compared with earlier results, and thus provides indications (warnings, alarms, reports) to themselves, their relatives, friends or carers.

These applications represent a core sample for making it possible to promote the architecture and create a business network from all sides of the Quadruple Helix, building a broad network both from the demand side, such as government institutions (health organizations) and from end-users (elderly as private persons) as well as from the supply side such as businesses like development consultancies or infrastructure companies. The initial offering of services is important in order to substantiate the possibilities of the platform as well as making a concrete impact on the solution area of "elderly living longer at home". 
During the framework development phase, the applications constitute both a way of making demands on the service platform and of proving how the platform could be effective as a development environment. At the same time, the platform creates a frame for application development, both as to limitations and as a source of technical innovation, providing possibilities for the developers. From a business model perspective, both platform and applications present opportunities to develop viable business networks.

\section{RESULTS}

This report aims at describing a project which realizes a Quadruple Helix setup, with the aim of transforming it into a long-term sustainable eHealth entity, where novel solutions can be developed, integrated and offered to elderly people living longer at home. To generalize this setup, a life-cycle logic is applied to frame the processes, the development of technical platforms and the delivery of applications. The core integrating process is seen as a cooperative design involving different groups of experts from all sides of the Quadruple Helix framework: public organization, users/civil society, business and research/education. The model (Figure 2) encapsulates the processes, the architecture and the resulting applications of Section 4, as described in the case. Here the interaction, knowledge creation and sharing which constitute the cooperative work come together in a holistic, lifecycle-based process.

The project suggests a major process innovation as a way of organizing an eHealth eco system. The working model consists of four major types of cooperation action phases, as these progress throughout the system's development life cycle; see Figure 2.

\subsection{Co-Investigation}

The aim of the "co-investigating" action is to create the principles behind formulating a framework for the application project. This includes establishing the main processes: project management, co-design, business planning and technical requirements analysis. In the eHealth area, this could mean, for example, the use of existing knowledge for evidence-based medicine and practice and include research in different scientific fields. The key activities comprise developing an understanding of the market and investigating potential partners and customers. Co-investigation includes a number of actions: co-planning, co-sensing and co-shaping with users and professionals for the creation and sharing of common understanding and knowledge. Its key outcomes are, for example, personal functional requirements and user motivations for actual usage. This is done by focusing on the selection of specific and limited use cases which are analyzed for the creation of design principles to be included in the general project framework.

\subsection{Co-Development}

The primary aim of co-developing is to develop a generic framework for the application. This includes the integration of end-to-end use cases and agile system development with co-creating and continued business planning. Different methods are applied including those for demonstrations, getting feedback from users and developing a first pilot for testing the framework. This first pilot is then co-explored by being tested in real life situations over a longer period of time and then being co-evaluated.

\subsection{Co-Implementation}

The primary aim of co-implementation is to develop a solution for a specific situation, which constitutes a process customizing the general application as developed from the preceding phase. In this phase, customers with specific demands co-implement the general solution. Hence, the framework needs to be customized to fit into the actual use cases, down to the individual level. This necessitates the creation of specific requirements and, if possible, integration into the customers' existing information systems. When new use cases are introduced, it becomes necessary to take a step back and make a rapid co-investigation and co-development for use cases that already exist and to match this with customers' specific circumstances and preferences. In this phase, the services (process changes, improvement work, implementation and support/maintenance processes) around the framework need to be defined and developed. 


\subsection{Co-Continued Development}

The major aim of co-continued development is to develop a sustainable application or prepare for its removal or replacement. To ensure good outcomes and continuous development in this phase, evaluation measurement instruments are needed, e.g. for the outcome in quality of life, for health status on a group level as well as for the level of use and technical performance. Continuous measuring will be the input for quality improvement work for both service and technical systems as well as for supporting costumers with their performance and enabling them to compare between solutions and require customizations.

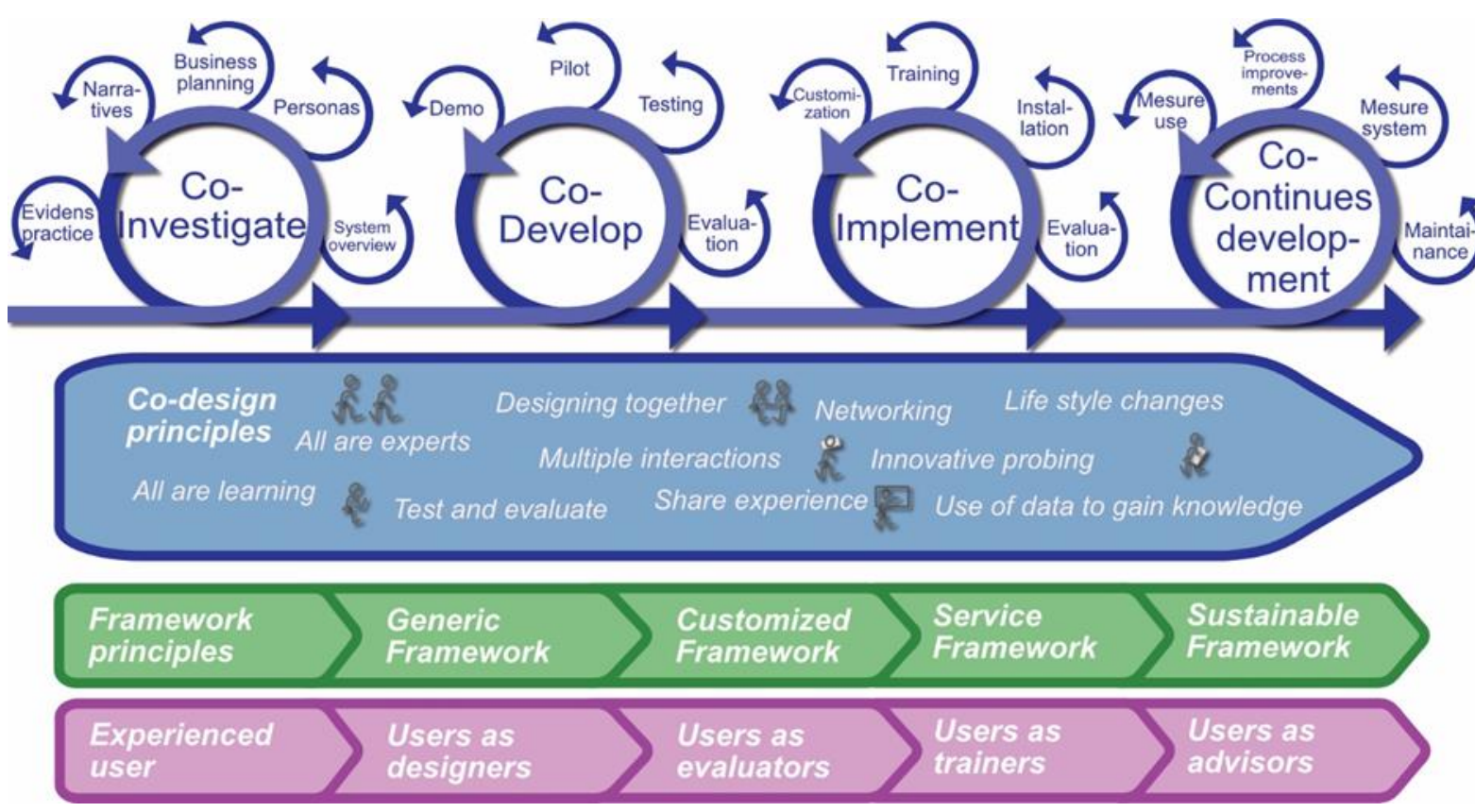

Figure 2. Quadruple Helix Co-Design Process

\section{CONCLUSIONS: FUTURE DEVELOPMENTS AND CHALLENGES}

At the core of a joint project lie the capabilities of cooperatively creating, sharing and deploying new knowledge. The project has worked with a set of core value statements guiding the cooperative processes. As illustrated in Figure 2, these include some key examples, like "all are experts", "sharing experience" and "all are learning", which all point to the primacy of the knowledge and learning dimensions. Contributing to the understanding and the conceptualization of action-oriented projects of this kind emerges as an important ongoing scientific undertaking. Further work into how to gain new knowledge about issues that are even broader than the sometimes narrow business or technical goals of development projects is important to make them more relevant to the big picture of the Quadruple Helix framework.

The end user holds a special position and role during the cooperative process, as marked by a purple arrow (Figure 2) illustrating different roles and responsibilities during the life cycle. This is a central tenet in the Quadruple Helix frame, as end users might be in a weaker position, especially in their roles as patients in the eHealth scenario. This is consistent with the addition made in the Quadruple Helix frame by the "media and culturally based public" to the triple helix of government, business and research. The fractured knowledge landscape is reflected in the core value of "all are experts" and makes room for the need of co-existence and co-evolution of the knowledge sets of a development project. Further research is required on how co-design concepts in a broad sense can form an approach to the operationalization of Quadruple Helix ideas, of which some initial findings have been shown here. Connecting the technological base (platforms) to innovative processes (how to organize) with the benefits of solutions (end user applications) through ideas of co-design emerges as one way forward. 


\section{ACKNOWLEDGEMENT}

This work has been performed within the project "FRONT-VL", (From empowerment to viable living, Front-vl.eu) with the support from the Vinnova, within the Celtic Next program.

\section{REFERENCES}

AARP/Roper Public Affairs \& Media group of NOP World, Beyond 50.05 Survey, 2004, https://assets.aarp.org/rgcenter/il/beyond_50_05_survey.pdf, Accessed 25 march 2019.

Carayannis, E.G. and Campbell, D.F.J. (2009). "'Mode 3' and 'Quadruple Helix': toward a 21st century fractal innovation ecosystem". Int. J. Technology Management. 46 (3/4): 201-234.

Carayannis, E.G., and Campbell, D.F.J. (2014) "Developed Democracies versus Emerging Autocracies: Arts, Democracy, and Innovation in Quadruple Helix Innovation Systems." Journal of Innovation and Entrepreneurship 3.1: 1-23.

Chevalier, J.M. and Buckles, D.J. (2013) "Participatory Action Research: Theory and Methods for Engaged Inquiry", Routledge UK.

Eisenhardt, K.M. (1989). "Building theories from case study research". Academy of Management Review, 14(4), pp. 532-550.

Foth, M. (2018). "Participatory urban informatics: Towards citizen-ability". Smart and Sustainable Built Environment, 7(1), 4-19.

Graybill EM, McMeekin P, Wildman J (2014) "Can Aging in Place Be Cost Effective? A Systematic Review". PLoS ONE 9(7): e102705. doi:10.1371/journal. pone.0102705

Hamm, J., Money, A., Atwal, A. \& Paraskevopoulos, A. (2016). "Fall prevention intervention technologies: A conceptual framework and survey of the state of the art". Journal of Biomedical Informatics, 59, 319-345.

Höglund, L. and Linton, G. (2017). "Smart specialization in regional innovation systems: a quadruple helix perspective". R\&D Management. 48 (1): 60-72.

Ivanova, I. (2014). "Quadruple Helix systems and symmetry: a step towards helix innovation system classification. Journal of the Knowledge Economy, 5, 357-369.

Khosravi, \& Ghapanchi. (2016). "Investigating the effectiveness of technologies applied to assist seniors: A systematic literature review". International Journal of Medical Informatics, 85(1), 17-26.

Leydesdorff, L. (2012). "The triple helix, quadruple helix, ..., and an N-tuple of helices: explanatory models for analyzing the knowledge-based economy?". Journal of the Knowledge Economy, 3, 25-35.

Miller, K., McAdam, R., Moffett, S., Alexander, A., and Puthusserry, P. (2016). "Knowledge transfer in university quadruple helix ecosystems: an absorptive capacity perspective". R\&D Management, 46, 383-399.

Mulder, I. (2018). "Co-creative partnerships as catalysts for social change”. Strategic Design Research Journal, 11(3), 178-185.

Roberts, C., Mort, M., \& Milligan, C. (2012). "Calling for Care: 'Disembodied' Work, Teleoperators and Older People Living at Home". Sociology: The Journal of the British Sociological Association, 46(3), 490-506.

Sanders, E.B.N. Stappers P.J. (2008). "Co-creation and the new landscapes of design”. CoDesign, 4(1) pp. 5-18

Sixsmith, A. and Sixsmith J. (2008). "Ageing in Place in the United Kingdom”. Ageing Int., 32:219-235.

Stake .E. (1995). "The art of case study research". Sage, Thousand Oaks, CA.

Suchman, L. (1993). “Forward”. In; (eds.) D. Schuler and A. Namioka: Participatory Design: Principles and Practices. CRC Press.

Turjamaa, R., Hartikainen, S., Kangasniemi, M. and Pietilä, A. (2014). "Living longer at home: a qualitative study of older clients' and practical nurses' perceptions of home care". J Clin Nurs, 23: 3206-3217. 